\title{
APMA Database for Affymetrix Target Sequences Mapping, Quality Assessment and Expression Data Mining
}

\author{
Yuriy Orlov ${ }^{1}$, Jiangtao Zhou ${ }^{1}$, Joanne Chen ${ }^{2}$, Atif Shahab ${ }^{1,2}$, \\ and Vladimir Kuznetsov ${ }^{1, *}$ \\ ${ }^{1}$ Genome Institute of Singapore, 60 Biopolis Street, Genome, \\ 138672 Singapore \\ ${ }^{2}$ Bioinformatics Institute, 30 Biopolis Street, Matrix, \\ 138671 Singapore \\ kuznetsov@gis.a-star.edu.sg
}

\begin{abstract}
We have developed an online database APMA (Affymetrix Probe Mapping and Annotation) for interactive presentation, search and visualization of Affymetrix target sequences mapping and annotation <http://apma.bii.astar.edu.sg >. APMA contains revised genome localization of the Affymetrix U133 GeneChip initial (target) probe sequences. We designed APMA to use it as a filter before data analysis and data mining so that noise expression signals, false correlations and false gene expression patterns can be reduced. Discrepancies found in probeset annotation and target sequence mapping account for up to $30 \%$ of probesets, including about $25 \%$ of Affymetrix probesets derived from target sequences overlapped interspersed repeats and $1.8 \%$ of original target sequences with erroneous orientation of the sequences. $86 \%$ of U133 target sequences passed our quality-control filtering.
\end{abstract}

Keywords: Affymetrix U133, database, target sequences, cross-hybridization, mapping, genome repeats, errors, classification, recognition, data mining.

\section{Introduction}

The increasing growth of the microarray researches demands high quality standards for microarray expression databases, description and annotation of probes and genes. One of the key problem facing microarray experiments is insufficient reliability of expression measurements due to sub-optimal probe design. The problem could originate from poor gene identification by the probe sequences, whose design may not consider the actual complexity of the human transcriptome. Poor quality control (QC) of microarray probes can also generate many hard statistical problems at data analysis level, starting from selection of differentially expressed genes and ending by identification of co-expressed and co-regulated genes.

One of the widely accepted microarray technologies is provided by Affymetrix Corporation (http://www.affymetrix.com). Our goal was to develop an algorithm and

\footnotetext{
${ }^{*}$ Corresponding author.
} 
software for quality control and filtering of Affymetrix target sequences. We organized and stored the results of this work in the APMA (Affymetrix Probe Mapping and Annotation) database. Such database suggested is of reasonable practical interest of the users of Affymetrix microarrays.

In situ synthesized oligonucleotide Affymetrix GeneChip uses a set (the so called probeset) of 11-20 oligonucleotide probes, each 25 bases long, to represent a gene or a gene transcript. The perfect match probe comes together with a mismatch probe designed to measure non-specific cross-hybridization. The expression level for a gene is a summary of the signal from the entire probeset. Affymetrix uses $\sim 150-450 \mathrm{nt}$ initial (target) sequences of genes for probes (and whole probeset) location.

The problem of accurate Affymetrix target sequence annotation is related to the complexity of multiple "gene models" including unverified ESTs from public datasets. Reported re-identification of genes may affect $30-50 \%$ of probesets $[1,2]$. Recent papers $[3,4,5,6]$ report re-evaluation of Affymetrix microarray probes using BLAST comparison of probe sequences to the complete human genome. In some cases, multiple probesets can specifically target a single genic sequence coding for protein. In other cases, however, a probeset is capable of hybridizing to more than one transcript (and provide uncertainty in transcript detection) [7].

Selection of original target sequences is one of the key steps of probe design process. There are several basic quality control criteria for verification of the target sequence. The sequences should: (1) detect a unique locus in human genome, (2) match a single transcript without mutations (correct mapping); (3) correspond to the sequence from the transcribed strand of the genome at the locus (correct strand orientation of target sequence); (4) not overlap with any other non-gene sequence that could cross-hybridize or even be independently transcribed (segmental duplications, interspersed repeats); (5) correspond to mature RNA (not intronic sequences that are spliced).

Unfortunately, these basic criteria have not been well controlled. Perhaps, this is the case because transcript databases are incomplete, contain erroneous sequences, and undergo continual growth and change. Figure 1 shows examples of poor designed Affymetrix target sequences.

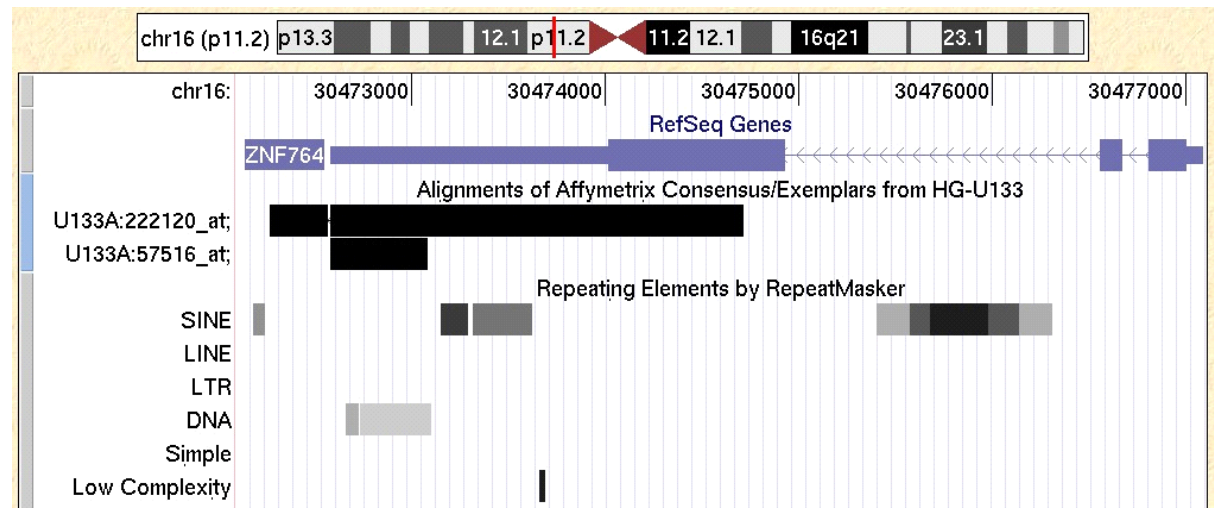

Fig. 1. Examples of problematic Affymetrix target sequences. Target sequence A.222120_at corresponds to ZNF764 gene and contains SINE and DNA repeat elements. Target sequence A.57516_at falls completely (95\%) into repeat element (Charlie 8, DNA/MER1 type). The last probeset evidently has great potential for cross-hybridization. 
Target sequence A.222120_at maps only one exon of ZNF764 gene (Fig. 1). It contains repeats (SINE and Alu) that could cause cross hybridization of probes. Target sequence A.57516_at overlap DNA/MER1 repeat by $95 \%$ and definitely has great potential for cross-hybridization and biased measuring of expression signal for this gene.

In our previous studies [8,9], we have developed software for automatic annotation and quality control of U133A and U133B targets sequences. In this work, we focus on data basing, QC of target sequences U133 Plus 2.0 GeneChip and using these tools for characterization of erroneous patterns in target sequences and expression data sets. We use BLAT program for target sequence mapping to check correspondence of probesets to annotated genes. We develop online database for interactive presentation, search, filtering and visualization of Affymetrix U133 Plus 2.0 target sequences and their mapping and annotation. The database collects information on erroneous probesets and provides flexible filters for pre-processing expression data. Finally we use several large cancer cell expression data sets to estimate quality of unreliable target sequences and corresponding probesets.

\section{Methods}

Affymetrix sequence data for the U133A and U133B GeneChips were downloaded from the NetAffx web site (http://www.affymetrix.com/analysis/index.affx). These sequences, intended to represent genes, are referred to as initial target sequences of the Affymetrix probesets. We used BLAT search at $90 \%$ similarity level to match each Affymetrix target sequence to the genome. Then, we annotated overlaps with exonic region(s) of RefSeq, mRNA and spliced EST variants on the NCBI Build 35 and 36.1 (hg17 and hg18) assemblies. Example of target sequences annotation in APMA is in Figure 2.

\begin{tabular}{|c|c|c|c|c|c|c|c|c|c|}
\hline ApMA Alig & anme & nt -full & rersio & & & & & & \\
\hline Library: U & $133 s$ & et_upd & ated . & p1 & & & & & \\
\hline Results 1 to 50 & 0 of 42 & 08: K & 4 & Page $\longdiv { 1 }$ & & & & & \\
\hline Show Annotati & ions: & $\Gamma \mathrm{R}$ & fSeq & $\Gamma$ Known Ge & & $\Gamma$ Genbank mRNA & $\Gamma$ Spliced & & Go \\
\hline$\frac{\text { Chromosome }}{\text { Bin }}$ & Index & Affy ID & Strand & $\begin{array}{c}\text { Chromosome } \\
\text { Location(Start-End) }\end{array}$ & \begin{tabular}{|l|} 
Best Track \\
Annotation
\end{tabular} & Block Locations & Block Sizes & $\underline{\text { Span }}$ & \% Identity \\
\hline 6.0 & 1 & $1007 \mathrm{~s}$ at & + & chr6:30975289-30975862 & $\begin{array}{l}\text { Spliced } \\
\text { EST }\end{array}$ & {$[30975289,30975862]$} & 573 & 573 & 99.30 \\
\hline 7.0 & 2 & 1053 at & - & chr7:73094596-73090948 & $\begin{array}{l}\text { Spliced } \\
\text { EST }\end{array}$ & $\begin{array}{l}{[73090948,73091197]} \\
{[73094512,73094596]}\end{array}$ & 249,84 & 3648 & 98.78 \\
\hline 1.0 & 3 & 117 at & + & chr1:158308953-158309398 & $\begin{array}{l}\text { GenBank } \\
\text { mRNA }\end{array}$ & [158308953,158309398] & 445 & 445 & 100.00 \\
\hline 2.0 & 4 & 121 at & - & chr2:113691692-113691169 & $\begin{array}{l}\text { GenBank } \\
\text { mRNA }\end{array}$ & [113691169,113691692] & 523 & 523 & 99.81 \\
\hline
\end{tabular}

Fig. 2. APMA database interface (http://apma.bii.a-star.edu.sg) 
We mapped Affymetrix probesets to gene sequence blocks based on the initial target sequences, not based on the individual 25-mers in the probe sets.

We checked for exonic repetitive elements using RepeatMasker. We constructed a table of repeats classified by family and repeat types (DNA, LTR, LINE, SINE, simple and low complexity repeats, etc.) indicating length of the Affymetrix target sequence covered by the each type of repeats.

Large fraction of Affymetrix target sequences maps to a transcript on an opposite strand. Substantial numbers of mRNAs and ESTs in cis-antisense loci represent natural anti-sense transcripts (NAST) derived from the opposite strand of the given (usually protein coding) gene [10]. In order to distinguish the Affymetrix target sequences matching NAST from the Affymetrix target sequences having wrong orientation at non-NAST loci, we developed a pipeline and constructed a local United Sense-Antisense Pairs (USAP) database [11]. The database annotates and classifies SA pairs by three annotation tracks (RefSeq, mRNA and EST sequences) and stores the information about SA genes supported by Affymetrix target sequences.

Expression data. To study functional usefulness of the problematic probes, we analyzed the expression patterns of Affymetrix probesets in 249 primary breast tumors (NCBI Gene Expression Omnibus (GEO) http://www.ncbi.nlm.nih.gov/geo/; data sets GSE4922). The cancer samples were split into groups by histologic grades corresponding to aggressiveness of breast cancer [12,13]. In addition, we used U133A\&B expression data from several normal and cancerous human brain tissues (GEO data sets GDS1962), and expression profiles representing lung cancer cell lines (GEO ID: GSE5816). MAS5 normalization was applied [14]. Then we performed global mean normalization to $\ln (500)$, which provides better consistency for a large fraction of expressed genes across microarrays.

Software. Our database interface is developed in Perl. For group comparison, MannWhitney U-test statistics were used for continuous variables and one-sided Fisher's exact test used for categorical variables (Statistica-6 and StatXact-6 software). We have also used SAM 3.1 (Statistical Analysis of Microarrays) software [15] to estimate the number of differentially expressed genes defined by Affymetrix probesets.

\section{APMA Database and Statistical Assessment of Probesets Quality}

The results of mapping (chromosome coordinates, orientation, details of overlapping with exons and repeats etc.) were stored in a local database associated with unique Affymetrix probesets ID (http://apma.bii.a-star.edu.sg/). The database has convenient user interface, search engine and visualization tools referring to external (Santa Cruz) and local (Singapore) versions of UCSC Genome Browser. The search engine allows to find annotation of Affymetrix ID by gene name or accession number. The interface is shown in Figure 3. 


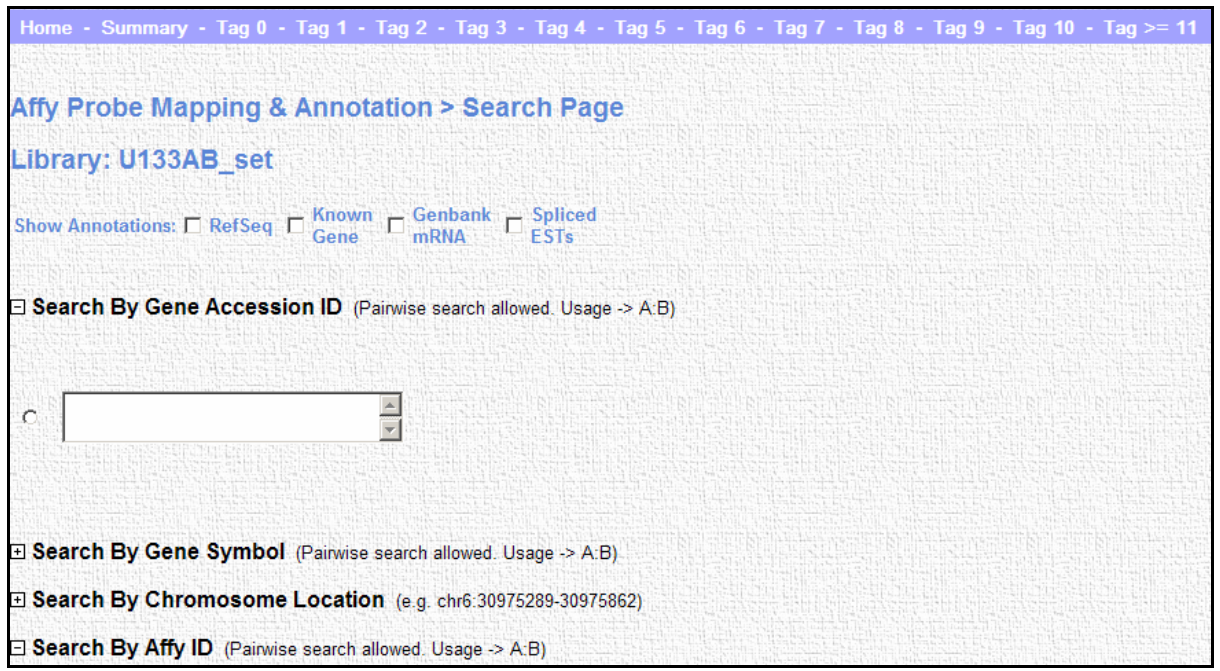

Fig. 3. Interface of the database search menu (http://apma.bii.a-star.edu.sg)

\subsection{Statistics of Problematic Groups of Target Sequences}

We believe that target sequences of purportedly human microarray probes which, by BLAT, are completely absent in the human genome (sequences to which we hereafter refer to as Tag0 sequences) and target sequences which match multiple loci in the genome (called Tag2, Tag3, etc. based on the number of their BLAT-matched loci) are sources of cross-hybridization effects in gene identification and should be excluded from analysis of microarray experiments. We checked BLAT mappings for all 44,692 sequences on U133A and B microarrays, except service and control probesets [16].

We found: (i) $1212(2.7 \%)$ initial target sequences which do not match any location in the human genome (Tag0 or mismatched sequences, see Tab. 1); (ii) 42708 $(95.5 \%)$ target sequences with a single reliable mapping (Tag1: reliable target sequences); (iii) 772 target sequences (1.7\%) with multiple locations in the human genome (Tag2+). Tag2 + is defined as sum of Tag2, Tag3, Tag4,.., etc. Tag 0 and Tag2+ might cause noise and/or cross-hybridization signals.

Table 1. Statistics of Affymetrix target sequence matches in human genome

\begin{tabular}{|l|l|l|l|}
\hline \#matches & \#Affymetrix ID & Percentage & Cumulative \% \\
\hline tag0 & 1212 & 2.71 & 2.71 \\
\hline tag1 & 42708 & 95.56 & 98.27 \\
\hline tag2 & 450 & 1.01 & 99.28 \\
\hline tag3 & 129 & 0.29 & 99.57 \\
\hline tag4 & 67 & 0.15 & 99.72 \\
\hline tag5+ & 126 & 0.28 & 100 \\
\hline Total & $\mathbf{4 4 6 9 2}$ & $\mathbf{1 0 0}$ & $\mathbf{1 0 0}$ \\
\hline
\end{tabular}


Tag0 sequences are related mostly to mRNA and EST, but not to genomic DNA. These sequences were associated with poorly-designed target sequences, poorlyannotated transcripts, and with nonhuman sequences which were mistakenly labeled as "human" in the GenBank. For instance, some of Tag0 were classified as "xenosequence /nonhuman" (mouse, cow, pathogens, rat etc; 224340_at is mouse c-Myc with extra TGA insertion; 217283_at strongly maps mouse short stature homeobox; 217255_at $100 \%$ is cow SQSTM1). Other probesets belong to small groups of poorlydefined sequences (for instance, 222196_at falls to random (not assembled) chromosome parts).

Standard assignment of Affymetrix target sequences to genome provided by UCSC Genome Browser using default BLAT parameters either does not define all target sequences or just skips them without any reference. Location of probesets could correspond to the mapping of genes, but the latter maybe not unique. (For example, target sequence for probeset 208303_s_at falls onto different chromosomes in hg18: $\mathrm{X}, \mathrm{Y}$ following the mapping of CRLF2 (cytokine receptor-like factor 2 isoform 1). The CDS end of the gene is not complete. Another example is 207353_s_at probeset mapped to the unassembled part of chromosome 4 (chr4_random).

We identified multiple genome locations of some extraordinary redundant probes (tag11+). For instance, probeset 81737_at has 22 different locations in human genome; probeset 213089_at has more 11 hits to human genome.

\subsection{Repeats in Tag1 Target Sequences}

Surprisingly, about $25 \%$ of target sequences are covered by mobile elements (repeats) abundant in the human genome such as Alu, LINE and LTR (Tab. 2).

They might serve as a significant source of erroneous detection of expressed genes and cross-hybridization signals.

Table 2. U133 Affymetrix target sequences containing genome repeats

\begin{tabular}{|l|l|c|c|}
\hline Set of repeats & Repeat class & $\begin{array}{l}\text { \# in U133A } \\
\text { and U133B }\end{array}$ & $\begin{array}{l}\text { \# in U133 } \\
\text { additional set }\end{array}$ \\
\hline Simple repeats & Simple repeat, Low complexity & 3233 & 468 \\
\hline Short transposons $(<300 \mathrm{bp})$ & DNA, SINE/Alu, SINE/MIR & 4347 & 1578 \\
\hline Long transposons $(>300 \mathrm{bp})$ & $\begin{array}{l}\text { LINE/CR1, LINE/L1, LTR/ } \\
\text { ERV1/ERVK/ERVL/MaLR }\end{array}$ & 5420 & 1915 \\
\hline $\begin{array}{l}\text { Non-transposons and } \\
\text { satellites }\end{array}$ & $\begin{array}{l}\text { Other, RNA, rRNA, scRNA, } \\
\text { Satellite, snRNA, srpRNA }\end{array}$ & 80 & 31 \\
\hline
\end{tabular}

\subsection{Inversely Oriented Target Sequences}

We consider an Affymetrix target sequence as inversely oriented if it matches the opposite strand to any RefSeq, mRNA, or EST-supported gene. If a target sequence matches also any RefSeq or mRNA in the same strand then this sequence may refer to natural antisense transcripts (NAST), but not annotation errors. We developed a pipeline to distinguish annotation errors from sequences matching natural antisense transcripts. We considered a target sequence as misoriented relative to the intended gene (presented by RefSeq or mRNA ID) if: 
1) it is aligned perfectly in complete genomic coordinates, block by block (the allowed shift is no more than $8 \mathrm{bp}$ except for the leftmost and rightmost block) to the transcript mapped to the opposite DNA strand;

2) the number of blocks RefSeq/mRNA blocks mapped to the genome was greater than one;

3) there no any RefSeq gene in the same strand;

4) if there are several perfectly matching mRNA transcripts in both strands target sequence matches the majority of GenBank mRNAs in wrong orientation, while there are none or only a single mRNA perfectly matching the Affymetrix target sequence blocks on the same strand.

In total, $810(1.8 \%)$ Affymetrix target sequences were defined as misoriented target sequences. This set was identified by manual curation and automatic comparison of blocks of Affymetrix target sequences with exons of RefSeq or mRNA sequences in opposite strand (Tab. 3). The number of Affymetrix target sequences misoriented relative to intended transcripts is larger than previously reported by Harbig et al. [1].

\subsection{Classification of Different Categories of Problematic Affymetrix Target Sequences}

Tab. 3 shows the statistics of different categories of poorly-defined Affymetrix target sequences found using hg18 Assembly: Tag0, multiple genome matching Tag2+ (Tag2, Tag3, Tag4 and others) targets sequences, misoriented target sequences and the target sequences covered by genome repeats.

This table shows that only about 86\% (38511/44692) U133A\&B target sequences could be useful in expression analysis. Our pipeline identified 13260 Affymetrix target sequences matching SA gene pair loci. These target sequences match the natural SA transcripts and should not be excluded from the analysis.

Additionally, we have identified 810 erroneously oriented Affymetrix target sequences, which should be excluded from functional (expression) analysis.

Table 3. Joint classification of problematic Affymetrix GeneChip U133A\&B target sequences

\begin{tabular}{|l|l|l|}
\hline Target sequences groups & $\begin{array}{l}\text { Non-redundant \# of } \\
\text { probesets }\end{array}$ & $\%$ \\
\hline Total \# of non-Tag1 sequences, including: & 1984 & 4.43 \\
\hline Tag0 & 1212 & 2.71 \\
\hline Tag2+ & 772 & 1.72 \\
\hline Total \# of misoriented target sequences & 810 & 1.81 \\
\hline $\begin{array}{l}\text { Total \# of target sequences overlapped with repeats } \\
\text { including: }\end{array}$ & 3387 & 7.57 \\
\hline overlap 80-100\% of target sequence length & 761 & 1.7 \\
\hline Total \# of useful Tag1 sequences & 38511 & 86.16 \\
\hline TOTAL \# of Affymetrix target sequences & $\mathbf{4 4 6 9 2}$ & $\mathbf{1 0 0}$ \\
\hline
\end{tabular}




\subsection{Comparison of Mean Gene Expression Levels Detected by Different Classes of Problematic Target Sequences}

We compared average gene expression levels in the groups of problematic target sequences: tag0, multiple loci matching, misoriented relative to given gene, and target sequences covered by repeats by 40-60\%, 60-80\%, 80-100\% of target sequence length (in non-overlapping intervals of percents, i.e. [40;60), [60;80) and [80-100]) (Fig. 4). We used a large set of expression data of genetically and clinically well-separated breast cancer sub-types [12] for analysis of statistical parameters of the probesets. We designated Affymetrix probesets derived from target sequences without any complication or covered by genome repeats by less than $20 \%$ to $40 \%$ of target sequence length as "Normal". Fig. 4 shows strong negative trend of the mean values of hybridization signal from Normal to misoriented target sequences.

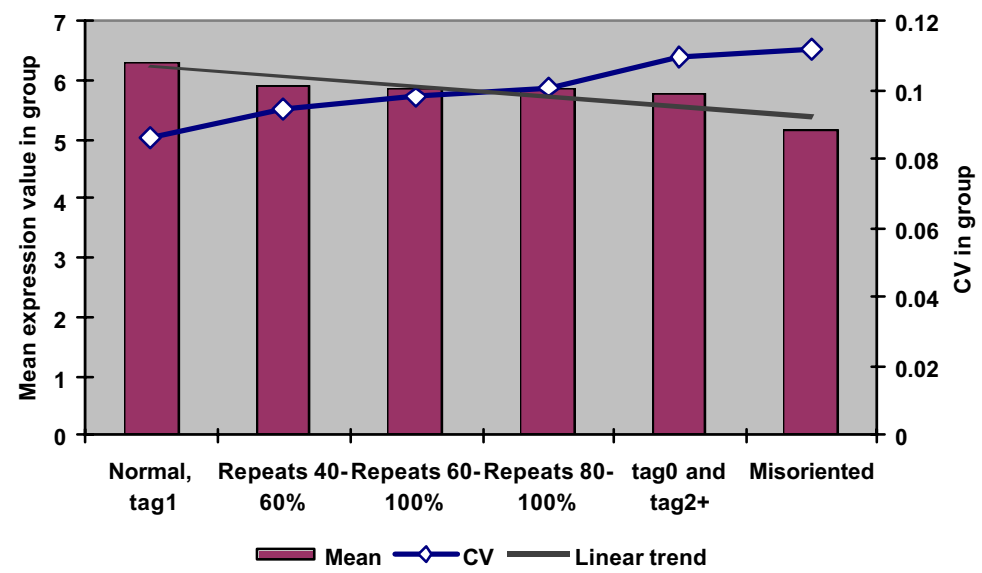

Fig. 4. Population average expression signal of probesets associated with problematic groups of Affymetrix target sequences. Columns present mean values in histologic Grade I of breast cancer samples for the probesets groups, line with diamonds presents corresponding coefficient of variation $(\mathrm{CV})$.

Misoriented and multiple-matching target sequences provide the poorest probesets in comparison with other problematic sequence groups. This trend is exhibited by the lower average expression signal and by the larger coefficient of variation $(\mathrm{CV})$.

We found that simple repeats and low-complexity sequences do not affect the ability of probesets to discriminate tumor-type specific signals [9]. However, as a general trend, target sequences with more genome repeats have progressively worsening proportions among differentially expressed genes in cancer tissue type comparison, especially for longer repeats (LTR and LINE) and for larger sequence span coverage of the target sequences.

Comparison of the numbers and values of correlation coefficients of probesets derived from multiple matching target sequences with random samples from Normal group reveals similarly poor quality of these problematic groups. Our analysis of 
expression data for cancer samples reveals that larger number of genome loci for the target sequence correlates with 1) higher expression noise (defined by CV-value), 2) lower average signal level, and 3) higher number of spurious positive correlations. This is what we would expect due to nonspecific hybridization signals.

\subsection{Comparison of U133A, U133B and Additional to U133 Plus2.0 GeneChips}

Figure 5 shows that the averages of signal intensity values for brain cancer cell samples differ for probesets from Normal group and probesets from "problematic" group. Problematic probesets have lower signals and therefore are enriched in the left (noisy-like) part of the empirical signal intensity frequency distribution (Fig. 5). Inversely, the signal value of Normal probesets is much enriched in the right part of the empirical signal intensity frequency distribution.

We have observed that the microarrays U133A and U133B show a markedly different quality of the target sequences and, respectively, of the hybridization signals of the probesets presented on these microarrays. Tab. 5 shows that the fraction of target sequences that passed our QC (quality control, i.e. Tag1, correct orientation on chromosome, and repeat coverage is less than $40 \%$ of target sequence length) on microarray U133A is larger in comparison to microarray U133B. In general, microarray U133A is better annotated and as we have showed exhibits higher expression level of genes than microarray U133B. We have observed $89.3 \%$ Normal target sequences for microarray U133A and $83 \%$ of such sequences for microarray U133B. Additional set (9983 probesets) to microarray U133 Plus 2.0 exhibits $79.6 \%$ of such non-problematic target sequences).

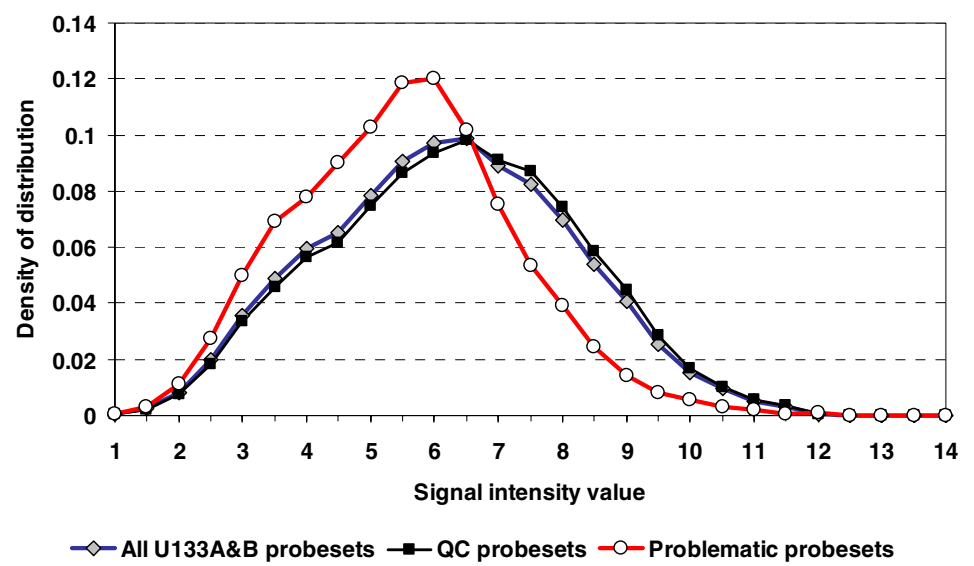

Fig. 5. Comparison of the histograms of signal intensity value for unfiltered (diamond), Normal filtered (square) and problematic (circle) probesets demonstrate systematic shift of the frequency of signal intensity value. Normal probesets filtered out based on quality control (QC) criteria show relatively higher signals. MAS5 normalized and log-transformed signals of brain cancer cell data set were used. 
Table 4. Target sequences passed QC for U133A, U133B and additional to U133 Plus 2.0 microarrays

\begin{tabular}{|l|l|l|l|}
\hline & $\begin{array}{l}\text { \# target } \\
\text { sequences }\end{array}$ & $\begin{array}{l}\text { \# target sequences } \\
\text { passed QC }\end{array}$ & $\begin{array}{l}\text { target sequences } \\
\text { passed QC, \% }\end{array}$ \\
\hline Common for A and B chip & 100 & 98 & 98.0 \\
\hline U133A & 22115 & 19753 & 89.3 \\
\hline U133B & 22477 & 18660 & 83.0 \\
\hline Additional to U133 Plus 2.0 & 9983 & 7942 & 79.6 \\
\hline
\end{tabular}

There are many examples of significant distinguishing expression levels of probesets, which corresponding to the same gene but are expressed differently on these three U133 sets. The poor-quality target sequences used to design the probesets (designed often using incomplete mRNAs or unreliable EST sequences) should be excluded from the gene expression analysis. Our database allows making this procedure automatically.

\section{Discussion and Conclusion}

Careful analysis of microarray probe design should be an obligatory component of MicroArray Quality Control (MACQ) project [17] initiated by the FDA USA in order to provide quality control tools to researchers of gene expression profiles and to translate the microarray technology from bench to bedside. In particular, identifying and filtering of unreliable target sequences are important data preprocessing steps before any analysis of microarray expression data. Such search and making decision strategy may provide essential improvement in selection of differentially expressed genes, gene clustering and pattern recognition of genetic and clinical subtypes, and in construction of realistic co-regulatory expression networks.

In this study, we have i) revised genome localization of the Affymetrix U133 GeneChip initial target sequences, ii) evaluated the impact of erroneous and poorly annotated target sequences on the quality of gene expression data and iii) developed an online database for interactive presentation, search and visualization of Affymetrix target sequences mapping and annotation. This DB contains revised genome localization of the Affymetrix U133 GeneChip initial (target) probe sequences.

In many cases, the spurious correlations can lead to serious erroneous interpretation of the microarray results, as was shown in [18]. In performing an analysis of overlaps of Affymetrix target sequences with repeat elements, we have quantitatively demonstrated that the number of positive correlation coefficients between genes in such a type of problematic target sequence group increases as repeat coverage increases. These extra false correlations in the groups do not correspond to real gene co-regulation but solely to bad design of target sequences. Similarly, Tag2+ and Tag0 can be also a significant source of spurious correlations of signals from probesets (and representative genes) on microarrays. Nevertheless researchers use such correlations without suitable quality control. Moreover, some gene discovery methodologies, such as hierarchical clustering, principal component analysis and gene-networking use the correlation coefficient of expression signal values between 
probesets as basic information. Other analyses, for example, general linear models, also are ultimately based on correlation-like principles.

Multiple-locus, nonhuman, misoriented, and nonspecific targets sequences are a significant attribute of the U133 GeneChip probesets. The ability of probesets to hybridize to more than one gene product can lead to false positives when analyzing gene expression data. The apparent artifacts in the data exist because the original target sequence annotations do not accurately correspond to the transcripts. Identification and removal of inaccurate target sequences can significantly improve specificity of GeneChip technology.

We summarize that about 14\% of U133 A\&B Affymetrix probesets have been designed based on erroneous target sequences. This fraction can be further classified as follows:

$2.7 \%$ of target sequences do not reliably match any location in the human genome;

Another $1.7 \%$ of the sequences have multiple locations (up to 10 times and more);

About $7.5 \%$ of the remaining Affymetrix target sequences overlap repeat elements abundant in the human genome completely including target sequences located in transposons or over more than $40 \%$ of the target sequence length, yielding noisy expression signal;

$1.8 \%$ of Affymetrix probesets have wrong orientation relative to the transcript they are alleged to detect.

The concrete number of the filtered out problematic Affymetrix probesets could be refined depending on the stringency of criteria. Despite numerous wrongly designed and poorly annotated target sequences, we argue that Affymetrix U133 GeneChip could show reproducible and quantitative hybridization signals. However, about $14 \%$ of these signals need filtering based on our expression analysis criteria, genome reannotation and statistical methods described in this paper. We suggest restricting data analysis and data mining of Affymetrix U133 probesets within the Normal artifactfree Tag1 probes with minimal repeat content.

In conclusion, we suggest that the development of APMA DB and computational QC tools could be used as an integrative filter system to be applied before data analysis and data mining in order to reduce noise expression signals, false correlations and false gene expression patterns.

Acknowledgments. Authors are grateful to Joshy George, Leonard Lipovich, Yong How Choong, Caleb Khor, Leong Cheok and Chuah Yuxin for help in processing of Affymetrix data and discussions as well as to A*STAR for support of the research.

\section{References}

1. Harbig, J., Sprinkle, R., Enkemann, S.A.: A sequence-based identification of the genes detected by probesets on the Affymetrix U133 plus 2.0 array. Nucleic Acids Res. 33(3), e31 (2005)

2. Okoniewski, M.J., Miller, C.J.: Hybridization interactions between probesets in short oligo microarrays lead to spurious correlations. BMC Bioinformatics 7, 2761 (2006)

3. Mecham, B.H., Wetmore, D.Z., Szallasi, Z., Sadovsky, Y., Kohane, I., Mariani, T.J.: Increased measurement accuracy for sequence-verified microarray probes. Physiol Genomics 18, 308-315 (2004) 
4. Gautier, L., Moller, M., Friis-Hansen, L., Knudsen, S.: Alternative mapping of probes to genes for Affymetrix chips. BMC Bioinformatics 5, 111 (2004)

5. Leong, H.S., Yates, T., Wilson, C., Miller, C.J.: ADAPT: a database of affymetrix probesets and transcripts. Bioinformatics 21, 2552-2553 (2005)

6. Dai, M., Wang, P., Boyd, A.D., Kostov, G., Athey, B., Jones, E.G., Bunney, W.E., Myers, R.M., Speed, T.P., Akil, H., Watson, S.J., Meng, F.: Evolving gene/transcript definitions significantly alter the interpretation of GeneChip data. Nucleic Acids Res. 33, e175 (2005)

7. Stalteri, M.A., Harrison, A.P.: Interpretation of multiple probe sets mapping to the same gene in Affymetrix GeneChips. BMC Bioinformatics 15, 8-13 (2007)

8. Orlov, Y.L., Zhou, J.T., Lipovich, L., Yong, H.C., Li, Y., Shahab, A., Kuznetsov, V.A.: A comprehensive quality assessment of the Affymetrix U133A\&B probesets by an integrative genomic and clinical data analysis approach. In: Kolchanov, N.A. (ed.) Proceedings of the Fifth International Conference on Bioinformatics of Genome Regulation and Structure, Novosibirsk, Inst. of Cytology\&Genetics, vol. 1, pp. 126-129 (2006)

9. Orlov, Y.L., Zhou, J., Lipovich, L.L., Shahab, A., Kuznetsov, V.A.: Quality assessment of the Affymetrix U133A\&B probesets by target sequence mapping and expression data analysis. In: Silico Biol. (in press)

10. Zhang, Y., Liu, X.S., Liu, Q.R., Wei, L.: Genome-wide in silico identification and analysis of cis natural antisense transcripts (cis-NATs) in ten species. Nucleic Acids Res. 34, 3465 3475 (2006)

11. Kuznetsov, V.A., Zhou, J.T., George, J., Orlov, Y.L.: Genome-wide co-expression patterns of human cis-antisense gene pairs. In: Kolchanov, N.A. (ed.) Proceedings of the Fifth International Conference on Bioinformatics of Genome Regulation and Structure, Novosibirsk, Inst. of Cytology\&Genetics, vol. 1, pp. 90-93 (2006)

12. Ivshina, A.V., George, J., Senko, O.V., Mow, B., Putti, T.C., Smeds, J., Lindahl, T., Pawitan, Y., Hall, P., Nordgren, H., Wong, J.E., Liu, E.T., Bergh, J., Kuznetsov, V.A., Miller, L.D.: Genetic reclassification of histologic grade delineates new clinical subtypes of breast cancer. Cancer Res. 66, 10292-10301 (2006)

13. Chua, A.L.-S., Ivshina, A.V., Kuznetsov, V.A.: Pareto-Gamma Statistics reveals global rescaling in transcriptomes of low and high aggressive breast cancer phenotypes. In: Rajapakse, J.C., Wong, L., Acharya, R. (eds.) PRIB 2006. LNCS (LNBI), vol. 4146, pp. 49-59. Springer, Heidelberg (2006)

14. MAS 5.0 algorithm. Statistical Algorithms Description Document. Santa Clara, CA: Affymetrix, Inc. (2002), http://www.affymetrix.com/support/technical/whitepapers/saddwhitepaper.pdf

15. Tusher, V.G., Tibshirani, R., Chu, G.: Significance analysis of microarrays applied to the ionizing radiation response. Proc. Natl. Acad. Sci. USA 98, 5116-5121 (2001)

16. Liu, G., Loraine, A.E., Shigeta, R., Cline, M., Cheng, J., Valmeekam, V., Sun, S., Kulp, D., Siani-Rose, M.A.: NetAffx: Affymetrix probesets and annotations. Nucleic Acids Res. 31, 82-86 (2003)

17. Shi, L., Reid, L.H., Jones, W.D., et al.: The MicroArray Quality Control (MAQC) project shows inter- and intra-platform reproducibility of gene expression measurements. Nat. Biotechnology 24, 1151-1161 (2006)

18. Lu, X., Zhang, X.: The effect of GeneChip gene definitions on the microarray study of cancers. Bioessays 28, 739-746 (2006) 